\title{
A Risk Calculator for Post-Operative Urinary Retention (POUR) Following Vaginal Pelvic Floor Surgery: Multivariable Prediction Modelling
}

\author{
Breffini Anglim ${ }^{1}$, George Tomlinson ${ }^{1}$, Joalee Paquette ${ }^{1}$, and Colleen McDermott ${ }^{1}$ \\ ${ }^{1}$ University of Toronto
}

January 5, 2022

\begin{abstract}
Objective: To determine the peri-operative characteristics associated with an increased risk of post-operative urinary retention (POUR) following vaginal pelvic floor surgery. Design: A retrospective cohort study using multivariable prediction modelling. Setting: A tertiary referral urogynaecology unit. Population: Patients undergoing vaginal pelvic floor surgery from January 2015 to February 2020. Methods: Eighteen variables (24 parameters) were compared between those with and without POUR and then included as potential predictors in statistical models to predict POUR. The final model was chosen as the one with the largest c-index from internal cross-validation. This was then externally validated using a separate data set $(\mathrm{n}=94)$ from another surgical centre. Main Outcome Measures: diagnosis of POUR following surgery while the patient was in hospital. Results: Among the 700 women undergoing surgery, 301 (43\%) experienced POUR. Pre-operative variables with statistically significant univariate relationships with POUR included age, menopausal status, prolapse stage, and uroflow parameters. Significant peri-operative factors included estimated blood loss, amount of intravenous fluid administered, operative time, length of stay, and specific procedures including vaginal hysterectomy with intraperitoneal vault suspension, anterior colporrhaphy, posterior colporrhaphy, and colpocleisis. The lasso logistic regression model had the best combination of internally cross-validated c-index (0.73) and accurate calibration curve. Using this data, a POUR risk calculator was developed (https://pourrisk.shinyapps.io/POUR/). Conclusions: This POUR risk calculator will allow physicians to counsel patients pre-operatively on their risk of developing POUR after vaginal pelvic surgery and help focus discussion around potential management options.
\end{abstract}

\section{Hosted file}

2022.01.01_POUR Manuscript_BJOG.docx available at https://authorea.com/users/454144/articles/ 551858-a-risk-calculator-for-post-operative-urinary-retention-pour-following-vaginalpelvic-floor-surgery-multivariable-prediction-modelling

\section{Hosted file}

2022.01.01_Tables and Figures_POUR Manuscript_BJOG.docx available at https://authorea.com/ users/454144/articles/551858-a-risk-calculator-for-post-operative-urinary-retentionpour-following-vaginal-pelvic-floor-surgery-multivariable-prediction-modelling 